\title{
Ligamentoplasty with interposition of the proximal interphalangeal joint in the treatment of unicondylar osteochondral defects: a cadaveric feasibility study
}

\author{
Jean-Charles Hery, Baptiste Picart, Mélanie Malherbe, Christophe Hulet, Aude Lombard \\ Department of Orthopedics and Traumatology, Caen University Hospital, Caen, France
}

Background Injuries to the proximal interphalangeal (PIP) joint are common and complex. However, the treatment of osteochondral defects of the head of the proximal phalanx has rarely been described. Herein, we propose a new technique for the management of unicondylar defects of the proximal phalanx that can restore joint amplitudes and provide PIP stability. Methods In this cadaveric feasibility study, unicondylar defects were generated using striking wedges and chisels. First, a transverse tunnel measuring $2 \mathrm{~mm}$ in diameter passing through the head of the proximal phalanx was made. A second tunnel at the base of the middle phalanx with the same diameter was then created. The hemitendon of the flexor carpi radialis graft was passed through each of these tunnels. The proximal end of the graft was interposed in the area with a loss of bone substance. The ligamentoplasty was then tensed and fixed by two anchors on the proximal phalanx. Joint amplitudes and frontal stability were measured preoperatively and postoperatively.

Results There was no significant change in the joint's range of motion: preoperatively, the mean mobility arcs were $-2^{\circ}$ to $113.80^{\circ}$, and they were $-2^{\circ}$ to $110^{\circ}$ after the procedure $(P=$ 0.999). There was no significant difference in joint stability $(P>0.05)$.

Conclusions Ligamentoplasty with PIP interposition appears to be a possible solution for the management of unicondylar defects of the proximal phalanx. An evaluation of clinical results is planned in order to definitively confirm the validity of this procedure.

Keywords Anatomy / Articular fracture / Finger / Collateral ligament reconstruction
Correspondence: Baptiste Picart Department of Orthopedics and Traumatology, Caen University Hospital, Avenue Cote de Nacre, 14000, Caen, France

Tel: +33-231-064-661

Fax: +33-231-065-303

E-mail: baptiste.picart@hotmail.fr

We would like to thank the team of the Faculty's Anatomy Laboratory.

\section{INTRODUCTION}

Injuries of the proximal interphalangeal (PIP) joint are very common $[1,2]$. These lesions can be accompanied by very complex osteocartilaginous lesions [3]. Reduction and fixation may be extremely difficult, or even impossible, for excessively large defects. The literature describes many surgical techniques for the management of osteochondral defects of the base of the middle phalanx (hemihamate grafting, costal grafting, mosaicplasty, etc.) [4-8]. The concept is to replace a missing section of the articular surface of the middle phalanx with an autologous osteochondral graft of similar geometry.

In comparison, the treatment of isolated defects of the proximal phalanx has been poorly described in the literature. Howev- 
er, the posttraumatic course of this type of fracture can move toward complete joint destruction, resulting in instability, subsequent deformity and significant loss of finger function [9].

Herein, we propose a ligamentoplasty method with interposition of a hemitendon of the flexor carpi radialis (hemi-FCR) for the management of unicondylar proximal phalanx osteochondral defects. This technique would have the advantage of filling the loss of bone substance through tendon interposition and strengthening the collateral ligament structure to reduce frontal laxity. The objectives were (1) to restore the joint amplitudes of the PIP and (2) to provide joint stability.

\section{METHODS}

In this cadaveric feasibility study, the second and third fingers were dissected on 20 hands (i.e., 40 PIPs). The other long fingers could not be used because there was not enough tendon graft available for four ligamentoplasties. The thumbs were not examined. Anatomical structures were similarly dissected using a dorsal approach.

\section{Surgical technique}

The incision was dorsolateral, curvilinear, and centered on the PIP. This incision enabled proper exposure for the rest of the procedure.

Unicondylar defects were carved with chisels and a gouge to generate osteocartilaginous defects that could be classified as London-Weiss I, IIa or IIb $[10,11]$. The hemi-FCR was prepared with the conventional procedure.

The first tunnel, with a diameter of $2 \mathrm{~mm}$, was made transversely through the head of the proximal phalanx, and then a second tunnel of the same diameter was made at the base of the middle phalanx. The tunnels were positioned at the insertion site of the main bundle of the collateral ligament. The intention was to preserve isometry in the flexion and extension of the ligament plane, thereby obtaining optimal frontal stability. Thus, for the proximal phalanx, the tunnel was made at the most proximal part of the osteochondral defect, parallel to the joint. For the middle phalanx, the tunnel was located $5 \mathrm{~mm}$ from the articular surface and palmar to the center line of the diaphysis (Fig. 1).

The tendon graft was inserted through each of the tunnels using a loop wire, as shown in Fig. 2. The proximal end of the graft was sutured to itself repeatedly to form an anchovy shape and then inserted into the area with loss of condylar substance to fill the cavity and thus obtain the required interposition. In a second step, a first 3.0 anchor (Mitek DePuy Synthes) was used to lock the graft to the proximal phalanx on the healthy side. The ligamentoplasty was then stretched and fixed by an anchor to

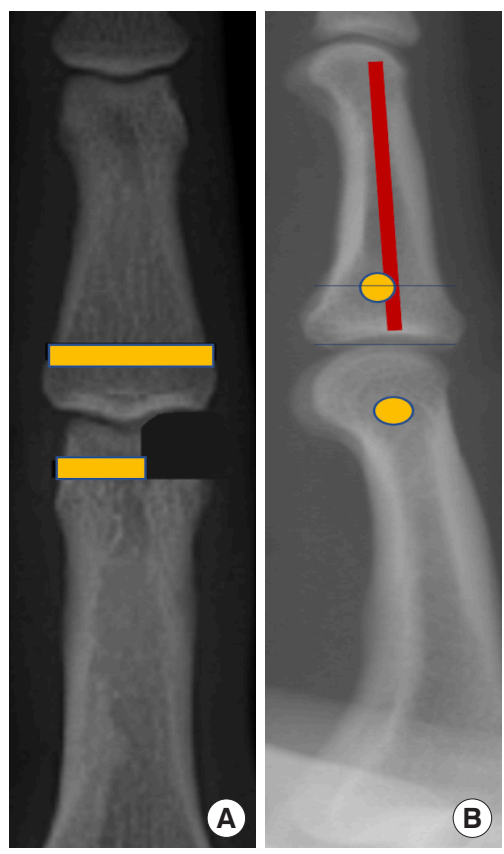

Fig. 1. Good positioning of the tunnels from the front $(A)$ and in profile (B). (A) The two orange lines represent the $2 \mathrm{~mm}$ tunnels on the proximal phalanx (lower) and the middle phalanx (upper). The tunnel was made at the most proximal part of the osteochondral defect, parallel to the joint. (B) The two orange spots represent the tunnel orifices on the proximal phalanx (bottom) and the middle phalanx (top). The red line corresponds to the axis of the diaphysis of the middle phalanx. The orifice of the tunnels is located palmar to the centerline of the diaphysis of the middle phalanx. For the distal phalanx, the tunnel is located $5 \mathrm{~mm}$ from the articular surface.

the proximal phalanx on the injured side (Fig. 3). The capsuloligament system and the skin incision were finally closed.

The joint amplitudes of the PIP were measured with a finger goniometer positioned on the dorsal side of the PIP. Frontal stability was subjectively estimated by applying the same varus-valgus strain on the PIP before and after the procedure.

\section{Statistical analysis}

Measurements performed on healthy PIPs were compared to those performed after surgery. A P-value $<0.05$ was considered to indicate statistical significance. The Wilcoxon test was used for qualitative variables and the Mann-Whitney test was used for quantitative variables. The software used was Excel (Microsoft Corp., Redmond, WA, USA) and SAS version 9.4 (SAS Institute Inc., Cary, NC, USA).

\section{RESULTS}

The healthy joints had an average range of motion of $-2^{\circ}$ to $113.80^{\circ}$ and were all stable before the procedure. After ligamentoplasty, the average angle was $110^{\circ}$ in flexion and $2^{\circ}$ in exten- 

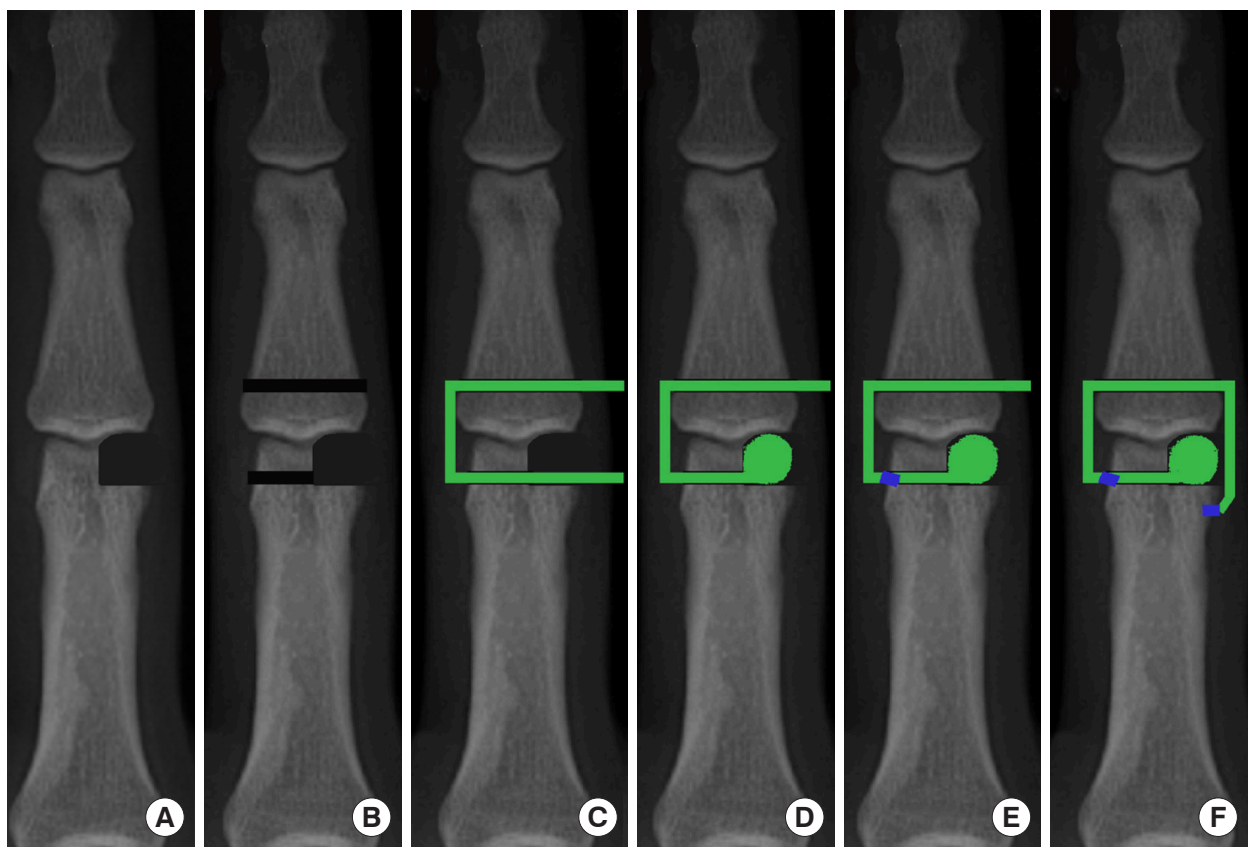

Fig. 2. Surgical technique plan. Illustration of the ligamentoplasty technique with interposition of the proximal interphalangeal (PIP) joint, step by step, from left to right (blue: surgical anchor and green: tendon graft). (A) Unicondylar defect of PIP. (B) Tunnels creation. (C) Passage of the graft through the tunnels. (D) Making the interposition. (E) First anchor positioning. (F) The graft is tensed to perform the ligamentoplasty and then locked with a second anchor.
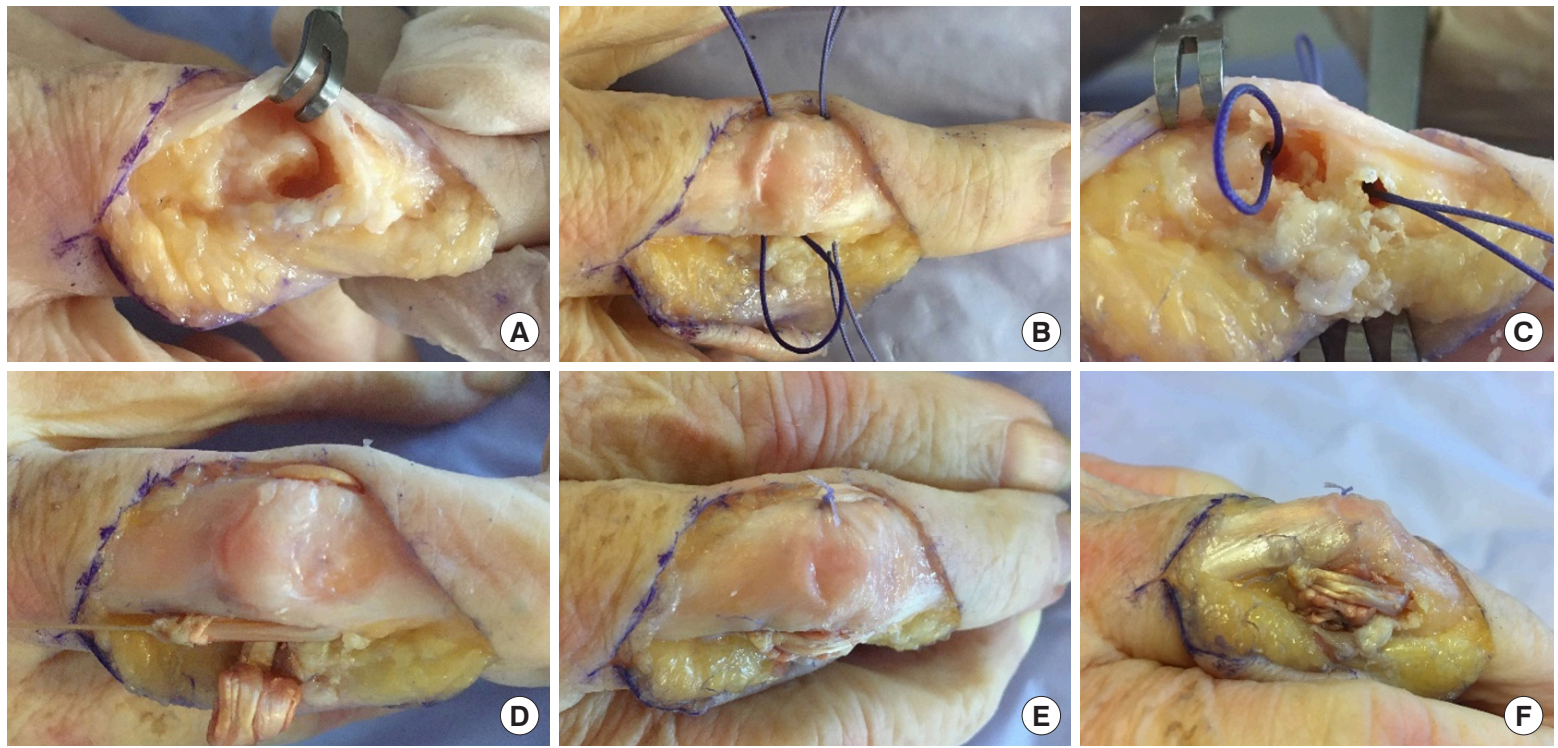

Fig. 3. Different steps of the surgical procedure. Unicondylar osteochondral defect London type 1 (A)-Loop allowing passage of the graft: dorsal (B) and fractured side views (C). Transplant passage (D)-final appearance: dorsal (E) and lateral views (F). Photographs taken with the authorization of the anatomy laboratory of the faculty of medicine.

sion (Table 1). In the 40 operated PIPs, no significant difference was found between postoperative and preoperative joint mobility $(\mathrm{P}=0.999)$.

After the procedure, $95 \%$ of the PIPs were stable. Stability was found in extension, as well as at $30^{\circ}$ and $90^{\circ}$ of flexion on the healthy and damaged sides (Table 2). The two cases of instabili-
Table 1. Range of motion of the proximal interphalangeal joint

\begin{tabular}{lccc}
\hline \multirow{2}{*}{ Range of motion } & \multicolumn{2}{c}{ Mean \pm SD } & \multirow{2}{*}{ P-value } \\
\cline { 2 - 3 } & Healthy joint $\left({ }^{\circ}\right)$ & Ligamentoplasty $\left({ }^{\circ}\right)$ & \\
\hline Flexion & $113.80 \pm 4.79$ & $110.00 \pm 4.24$ & 0.999 \\
Extension & $2.00 \pm 2.16$ & $2.00 \pm 1.20$ & 0.500 \\
\hline
\end{tabular}

Analysis of the range of motion of the proximal interphalangeal joint before and after the procedure. 
Table 2. Frontal stability testing

\begin{tabular}{lccc}
\hline Frontal stability & $\begin{array}{c}\text { Healthy joint } \\
(\mathrm{n}=40)\end{array}$ & $\begin{array}{c}\text { Ligamentoplasty } \\
(\mathrm{n}=40)\end{array}$ & P-value \\
\hline Healthy side & & & \\
In extension & 40 & 38 & 0.345 \\
At $30^{\circ}$ flexion & 40 & 38 & 0.345 \\
At $90^{\circ}$ flexion & 40 & 38 & 0.345 \\
Defect side & & & \\
In extension & 40 & 39 & 1.000 \\
At $30^{\circ}$ flexion & 40 & 38 & 0.345 \\
At $90^{\circ}$ flexion & 40 & 38 & 0.345 \\
\hline
\end{tabular}

Frontal stability between the healthy and defective sides before and after surgery in extension, at $30^{\circ}$ and $90^{\circ}$ of flexion.

ty were probably related to technical deficiencies. The first was probably due to the poor quality of the transplant. The second was the result of interposition that was too small and did not fill the entire bone defect. No significant difference in frontal stability was found between the two groups $(\mathrm{P}=0.345)$. Only two PIPs (5\%) showed postprocedural instability.

\section{DISCUSSION}

Defects of osteochondral substances in the PIP pose a surgical challenge. Reconstruction of the joint remains difficult and involves the risk of stiffness [12]. However, the PIP is acknowledged to be a key joint for overall finger function.

The technique proposed here, although it does not restore an anatomical joint surface, appears to restore satisfactory stability and mobility to the joint. Like ligamentoplasty and interposition after trapeziectomy, which provide excellent long-term results [13], this technique offers a twofold advantage: (1) it reduces the risk of deformation in the frontal plane by interposition of the tissue, and (2) it provides bilateral collateral reinforcement to support the interposition with ligamentoplasty. In addition, this technique enables the management of greater osteochondral substance loss than palmar plate advancement interposition arthroplasty which was initially described by Malerich and Eaton $[14,15]$. Furthermore, the two failures in this study illustrate the importance of combining ligamentoplasty and interposition. Ligamentoplasty with PIP interposition was performed using the anchovy technique, as was described for trapeziectomy filling by Burton and Pellegrini VD Jr [16]. The first clinical results of a technique combining interposition and collateral reinforcement were reported by Gong et al. [17]. Their technique combined interposition with tenodesis and reconstruction of the ipsilateral collateral ligament. The results of the three cases of posttraumatic destruction of the PIP treated by this technique showed, after an average of 2.5 years of followup, an average postoperative arc of mobility of $20^{\circ}$ to $60^{\circ}$, no pain, no mid-lateral instability, and an average grip strength of $88 \%$ compared with the healthy side. Moreover, this technique required minimal loss of osteochondral substance without significant joint incongruity.

A hemi-FRC graft was chosen for multiple reasons. This tendon has the advantages of being constant, and voluminous, ensuring that a graft can be harvested with the desired diameter and with a consequent length that enables filling of osteocartilaginous defects of all sizes and makes it possible to perform bilateral ligamentoplasty. Finally, we have experience harvesting these grafts for the management of other pathologies. This was not the case with the superficial common flexor, which is why it was not used.

For the construction of the tunnels, several diameters were tested before beginning this study. A realization of a 2-mm tunnel seemed to offer the best compromise between the passage of the transplant with a loop wire and the preservation of bone management. No fracture of the healthy condyle occurred during the protocol.

The use of two anchors on the proximal phalanx proved to be indispensable to guarantee the stability of the assembly. Indeed, the interposition alone did not allow self-locking that would be large enough to require only one anchor. For the same reason, and because their use is very frequent in hand surgery [18], anchors were preferred to tenodesis screws. Moreover, the latter could have weakened the healthy condyle of the proximal phalanx.

Two PIPs were found to be unstable postoperatively. The first was probably due to the poor quality of the transplant. Indeed, the transplant appeared to be damaged during the frontal stability test. The second was the result of an interposition that was too small and did not fill the full bone defect. Frontal instability increased with flexion (Table 2).

The choice of available treatments for osteochondral defects is limited. Arthrodesis is a reliable and simple technique, but stiffness is an obvious sequela, that is very poorly tolerated functionally [19]. Arthroplasty has not yet demonstrated acceptable results in young patients and may not be indicated in this type of trauma. Indeed, the complications inherent to this type of implant, such as instability, joint synovitis and prosthetic implant failure, make its use in young and active patients difficult; for this reason, no consensus exists regarding the use of these implants in such patients [20-22]. However, a recent study concluded that arthroplasty with a silicone implant is a viable option for acute management [23]. Vascularized toe joint transfer, or transfer of the distal interphalangeal joint of the same finger, has many advantages (composite transfer, satisfactory frontal stability), but it is technically challenging, creates morbidity at 
the donor site, exposes patients to microsurgical risks, and should probably only be considered in patients with loss of both joint surfaces [24-26]. Although they are poorly described, autologous osteochondral grafts are an attractive surgical option for restoring the lost segment of a joint surface. Ishida et al. [27] used the second or third carpo-metacarpal joint as a donor for osteochondral grafts for PIP reconstruction in 10 patients, with satisfactory results. Sato et al. [12] reported a significant improvement in joint mobility in nine PIP reconstructions with autologous costochondral grafts. Zappaterra et al. [28] confirmed these results in four patients with a mean arc of mobility of $20^{\circ}-50^{\circ}$. However, this donor area is at risk of pneumothorax, even though this risk is minimal when the correct approach is carried out. In 2010, satisfactory medium-term results were reported for a unicondylar reconstruction of the proximal phalanx using a graft taken from the base of the fifth metacarpal [29]. Recently, Kumta et al. [30] described the possibility of using microsurgical grafts from the femoral condyle for interphalangeal joint reconstruction.

There appear to be multiple advantages offered by the technique proposed here: (1) it makes it possible to preserve normal joint amplitudes; (2) it restores joint stability even in the event of significant osteochondral loss; (3) the transplant is known and has already demonstrated low morbidity; (4) the technique is simple to perform, is reproducible, and uses little material; and (5) this method does not prevent secondary arthroplasty if necessary. Nevertheless, this technique does present some difficulties, especially when positioning the bone tunnels and considering the existing risk of fracture of the healthy condyle during drilling. In addition, the management of graft isometry remains problematic. The best position for clamping the graft seems to be full extension, a position in which the collateral ligament plane is physiologically tense.

Questions remain as to the postoperative course and longevity of this surgical procedure. On the one hand, it will be important to evaluate the risks of collapse of the tendon interposition, relaxation of the graft and therefore secondary instability. On the other hand, an objective evaluation of frontal stability could not be performed due to the lack of measurement instruments. This remains the main limitation of this study. Therefore, it will be necessary to precisely measure frontal stability to obtain confirmatory clinical findings. Finally, to optimize functional results, it will be essential to develop a precise postoperative protocol, particularly concerning the type and duration of immobilization, as well as the rehabilitation to be implemented.

This technique is not indicated in cases involving loss of the bicondylar osteochondral substance and the middle phalanx base.
Ligamentoplasty with PIP interposition appears to be a possible solution for the management of proximal phalanx unicondylar defects. It has the potential to enable recovery of joint amplitudes and stability without compromising subsequent prosthetic replacement if necessary. An evaluation of the clinical results is envisaged to definitively validate this technique.

\section{NOTES}

\section{Conflict of interest}

No potential conflict of interest relevant to this article was reported.

\section{Author contribution}

Conceptualization: B Picart, A Lombard. Data curation: JC Hery. Methodology: B Picart, C Hulet. Visualization: M Malherbe. Writing - original draft: JC Hery. Writing - review \& editing: B Picart, M Malherbe, C Hulet, A Lombard.

\section{ORCID}

$\begin{array}{ll}\text { Jean-Charles Hery } & \text { https://orcid.org/0000-0002-0111-8997 } \\ \text { Baptiste Picart } & \text { https://orcid.org/0000-0003-1002-5431 } \\ \text { Mélanie Malherbe } & \text { https://orcid.org/0000-0001-7080-7350 } \\ \text { Christophe Hulet } & \text { https://orcid.org/0000-0002-1011-6141 } \\ \text { Aude Lombard } & \text { https://orcid.org/0000-0002-0490-4571 }\end{array}$

\section{REFERENCES}

1. Saitta BH, Wolf JM. Treating proximal interphalangeal joint dislocations. Hand Clin 2018;34:139-48.

2. Lee JJ, Park HJ, Choi HG, et al. Open reduction of proximal interphalangeal fracture-dislocation through a midlateral incision using absorbable suture materials. Arch Plast Surg 2013;40:397-402.

3. $\mathrm{Ng}$ CY, Oliver CW. Fractures of the proximal interphalangeal joints of the fingers. J Bone Joint Surg Br 2009;91:705-12.

4. Yamagami N, Yamamoto S, Tsujimoto Y, et al. Osteochondral autograft transplantation for malunited intra-articular fracture of the proximal interphalangeal joint: a case report. Arch Orthop Trauma Surg 2013;133:135-9.

5. Pillukat T, Kalb K, Fuhrmann R, et al. Reconstruction of the middle phalangeal base of the finger using an osteocartilaginous autograft from the hamate. Oper Orthop Traumatol 2019;31:393-407.

6. Williams RM, Kiefhaber TR, Sommerkamp TG, et al. Treatment of unstable dorsal proximal interphalangeal fracture/ dislocations using a hemi-hamate autograft. J Hand Surg Am 2003;28:856-65. 
7. Capo JT, Hastings H 2nd, Choung E, et al. Hemicondylar hamate replacement arthroplasty for proximal interphalangeal joint fracture dislocations: an assessment of graft suitability. J Hand Surg Am 2008;33:733-9.

8. McAuliffe JA. Hemi-hamate autograft for the treatment of unstable dorsal fracture dislocation of the proximal interphalangeal joint. J Hand Surg Am 2009;34:1890-4.

9. Hastings $\mathrm{H} 2$ nd, Carroll C 4th. Treatment of closed articular fractures of the metacarpophalangeal and proximal interphalangeal joints. Hand Clin 1988;4:503-27.

10. London PS. Sprains and fractures involving the interphalangeal joints. Hand 1971;3:155-8.

11. Weiss AP, Hastings $H$ 2nd. Distal unicondylar fractures of the proximal phalanx. J Hand Surg Am 1993;18:594-9.

12. Sato K, Sasaki T, Nakamura T, et al. Clinical outcome and histologic findings of costal osteochondral grafts for cartilage defects in finger joints. J Hand Surg Am 2008;33:511-5.

13. Rhee PC, Paul A, Carlsen B, et al. Outcomes of surgical management for thumb basilar arthritis in patients 55 years of age and younger. Hand (NY) 2019;14:641-5.

14. Malerich MM, Eaton RG. The volar plate reconstruction for fracture-dislocation of the proximal interphalangeal joint. Hand Clin 1994;10:251-60.

15. Eaton RG, Malerich MM. Volar plate arthroplasty of the proximal interphalangeal joint: a review of ten years' experience. J Hand Surg Am 1980;5:260-8.

16. Burton RI, Pellegrini VD Jr. Surgical management of basal joint arthritis of the thumb. Part II. Ligament reconstruction with tendon interposition arthroplasty. J Hand Surg Am 1986;11:324-32.

17. Gong HS, Chung MS, Oh JH, et al. Ligament reconstruction and tendon interposition for advanced posttraumatic arthritis of the proximal interphalangeal joint: 3 case reports. J Hand Surg Am 2008;33:1573-8.

18. Kim YW, Roh SY, Kim JS, et al. Volar plate avulsion fracture alone or concomitant with collateral ligament rupture of the proximal interphalangeal joint: a comparison of surgical outcomes. Arch Plast Surg 2018;45:458-65.

19. Pellegrini VD Jr, Burton RI. Osteoarthritis of the proximal interphalangeal joint of the hand: arthroplasty or fusion? J Hand Surg Am 1990;15:194-209.

20. Iselin F, Conti E. Long-term results of proximal interphalangeal joint resection arthroplasties with a silicone implant. J Hand Surg Am 1995;20(3 Pt 2):S95-7.

21. Lautenbach M, Kim S, Berndsen M, et al. The palmar approach for PIP-arthroplasty according to Simmen: results after 8 years follow-up. J Orthop Sci 2014;19:722-8.

22. Bravo CJ, Rizzo M, Hormel KB, et al. Pyrolytic carbon proximal interphalangeal joint arthroplasty: results with minimum two-year follow-up evaluation. J Hand Surg Am 2007; 32:1-11.

23. Laurent R, El Rifai S, Loisel F, et al. Functional evaluation following emergency arthroplasty of the proximal interphalangeal joint for complex fractures with silicone implant. Hand Surg Rehabil 2020;39:423-30.

24. Foucher G, Lenoble E, Sammut D. Transfer of a composite island homodigital distal interphalangeal joint to replace the proximal interphalangeal joint. Technique and case report. Ann Chir Main Memb Super 1990;9:369-75.

25. Foucher G, Lenoble E, Smith D. Free and island vascularized joint transfer for proximal interphalangeal reconstruction: a series of 27 cases. J Hand Surg Am 1994;19:8-16.

26. Hierner R, Berger AK. Long-term results after vascularised joint transfer for finger joint reconstruction. J Plast Reconstr Aesthet Surg 2008;61:1338-46.

27. Ishida O, Ikuta Y, Kuroki H. Ipsilateral osteochondral grafting for finger joint repair. J Hand Surg Am 1994;19:372-7.

28. Zappaterra T, Obert L, Pauchot J, et al. Post-traumatic reconstruction of digital joints by costal cartilage grafting: a preliminary prospective study. Chir Main 2010;29:294-300.

29. Cavadas PC, Landin L, Thione A. Reconstruction of the condyles of the proximal phalanx with osteochondral grafts from the ulnar base of the little finger metacarpal. J Hand Surg Am 2010;35:1275-81.

30. Kumta S, Warrier S, Jain L, et al. Medial femoral condyle vascularised corticoperiosteal graft: a suitable choice for scaphoid non-union. Indian J Plast Surg 2017;50:138-47. 